\title{
Resilience of High Voltage Transmission System
}

\author{
Naim H. Afgan ${ }^{1}$, Dejan B. Cvetinovic ${ }^{2}$ \\ ${ }^{1}$ Instituto Superior Tecnico, Lisbon, Portugal \\ ${ }^{2}$ Laboratory for Thermal Engineering and Energy, Institute of Nuclear Science "Vinca", \\ University of Belgrade, Belgrade, Serbia \\ E-mail:afgan@sbb.rs \\ Recieved March 2, 2011; revised April 20, 2011; accepted April 28, 2011
}

\begin{abstract}
The resilience of a system can be achieved by reducing its probability of failure as well as reducing the consequences from such failures and the time to recovery. Quantification of resilience is first approached from the broader societal context, from which the engineering sub-problem is formulated as an important building block of the integrated tool ultimately needed. Nonlinear structural responses are considered, as well as the impact of retrofit or repair. Impact on time to recovery is considered in all cases. The proposed framework makes it possible to relate probability functions, fragilities, and resilience in a single integrated approach, and to further develop general tools to quantify resilience. The high voltage transmission system is typical engineering system which requires the assessment of the resilience as the measure for evaluation of the potential hazard event development. In this respect the resilience of the high voltage transport system is highly vulnerable: central generation creates high value targets, long vulnerable transmission lines, unique high voltage transformers, vulnerable substations. The assessment of the resilience of the high voltage transmission system is based on the evaluation of the resilience index as the result of the sudden changes of the characteristic indicators.
\end{abstract}

Keywords: Sustainability, Resilience, High Voltage System, Resilience Indicators, Catastrophic Events

\section{Introduction}

Energy losses represents nowadays between $2 \%$ and $4 \%$ (depending of local climatic conditions) of total energy electric power transmission. In Europe, this figure is expected to grow as a result of the expected economic development of Southern and new EU states). For the case of the high voltage transmission sector, the energy losses depend on the temperature of the environment range. The high voltage system is highly vulnerable: central generation creates high value targets, long vulnerable transmission lines, unique high voltage transformers, vulnerable substations [1].

The electricity system currently experiences many disruptions due to natural hazards and human error. Large, costly blackouts occur frequently. It is evident that:

- It is highly vulnerable to human attack. A worst case scenario would be highly destructive.

- Many investments would simultaneously improve reliability \& reduce vulnerability or the amount of damage from terrorist attack.

- Evaluating the reliability and security benefits to- gether would justify many new investments.

In this analysis of energy grid system (Figure 1) the existing electricity distribution networks regulatory framework will be taken also into account. A Smart Grids Factor will be based on indicators such as grid volume and distributed generation rate. This factor is introducing the effect of regulation on the system, which is further to the performance-based ratemaking (guaranteed or overall standards). Using also the quality and efficiency factors implemented, a "third pillar" (besides economy and quality) for the regulation will be developed according to the countries existing incentive and quality based regulation [2].

This factor should reveal the current state of play, the changes compared to previous years and should allow a future outlook of the trends in network development. As an incentive it should have positive effects directly on the network operators' revenues in case of an improvement of the situation (less grid losses, more distributed generation compared to previous year). In order to measure the performance of each individual network operator and to make results comparable, each regulator should 


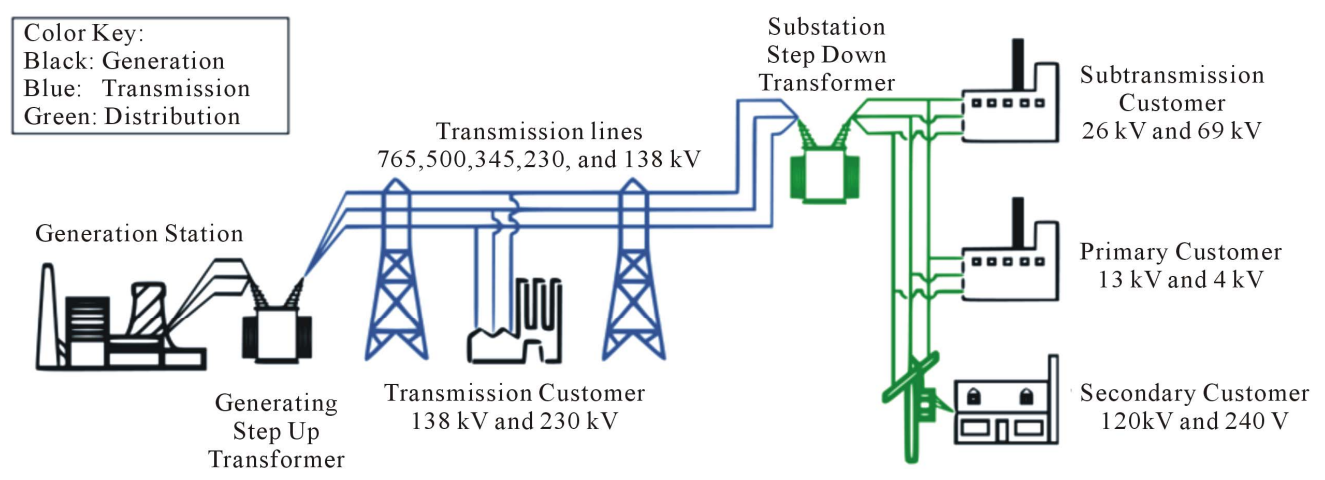

Figure 1. High voltage transmission system.

define reference values in advance. Different reference values for the individual operators are foreseen to include structural varieties. If the operator didn't reach the expected level it should consequently reduce its revenues. This would be an efficient method, which gives to network operators a financial incentive to foster network development in line with the approach of a Smart Grids $[3,4]$.

An electricity blackout causes us to freeze (sweat) in the dark. We find it difficult to: commute (no traffic signals, no trains); get up and down in buildings (no elevator); work (no light, computers, copiers, faxes); cook (no microwave, refrigerators, appliances, solid state ignition); get entertainment (no TV, radio, VCR). Almost all modern activities depend on electricity.

The high voltage system is highly vulnerable: central generation creates high value targets, long vulnerable transmission lines, unique high voltage transformers, vulnerable substations

System is disrupted frequently by natural hazards, human error, and human attack. Worst Cases Scenarios: Ice Storm: Quebec and NY in 1998, Hurricanes: Florida in 2004, Earthquake: Bay area, California in 1989. Hurricane Ivan: Almost occurred in 2004. Since these happened recently, a 500 year worst case would be much worse [5].

\section{Sustainability of High Voltage Transmission System}

Sustainability is the word which is used to create the special meaning for the interaction of the different entities in our world. In its definition sustainability was attributed to the interaction of system with its surrounding, including, social, cultural, environmental, economic and other aspects.

More than that, the sustainability has become a quality measure of the system in the assessment and evaluation of the respective system. It has been noticed that the sustainability comprise complexity definition for the com- plex system. In its definition the complex system is introduced as the nonlinear interaction of large number of elements functionally defined. Since, we can imagine a number of examples of complex systems in our life, it is of interest to verify some of them which are typical for the energy system. Complexity of the strategy of energy system is expressed through the multiple elements and their interaction [6].

The high voltage transmission system comprise a number of elements which functionality is defined in accordance with is role in the system. The complex system of high voltage transmission system is characterized with the specific number of the indicators reflecting individual properties of the system, as shown on Figure 2.

\section{Resilience Index for High Voltage Transmission System}

The sudden change of the indicator and its return to the primary state is the measurement of the capacity of the respective system to withstand the changes of the system. There are several potential changes of every system which may result in the eventual catastrophic event. It is of interest to visualize characteristic behavior following

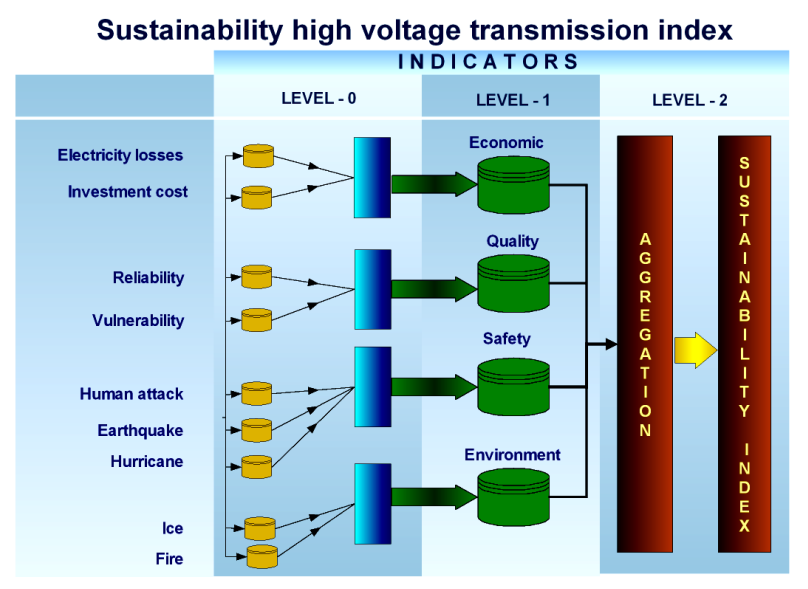

Figure 2. Sustainability high voltage transmission index. 
the sudden change of the indicator. Integral value of the indicator in the time scale until it reaches the steady state is the measuring parameter of the resiliency index [7-11].

Since the every sudden change of indicators may contribute to the resilience index, the sum of individual indicators of the sudden change as the resilience index is the value representing the capacity of the system under consideration. For the high voltage transmission system the Resilience Index is the agglomeration of capacity of the system reflecting the total change of the resilience capacity of the system $[12,13]$.

Figure 3 presents the sudden change of indicator value and its return to the steady state.

The agglomeration of the changes of all indicator represent the integral value of the Resilience Index expressed by Equation (1)

$$
R=\sum_{0}^{n} w_{n} \int_{t_{0}}^{t}\left(1-q_{n}\right)
$$

where

$w_{n}$-weighting coefficient;

$q_{n}$-indicator value in time scale;

$n$-number of indicators.

Figure 4 shows the Resilience Index monitoring scheme with procedure for the indicator agglomeration and presentation.

Definition of the Resilience Index can be simplified with the assumption that the integral format can be determined as the surface of the triangle formed by the amplitude of sudden change of indicator $\Delta q_{i}$ and time period $\Delta t_{i}$, Equation (2), so that

$$
R_{j}=w_{i} \sum_{0}^{n} \int_{t=t_{0}}^{t=t_{1}}\left[1-q_{i}(t)\right]=w_{i} \sum_{i=0}^{n} \frac{\Delta q_{i} \Delta t}{2}
$$

where

$\Delta q_{i}$-indicator change;

$\Delta t_{i}$ - time change.

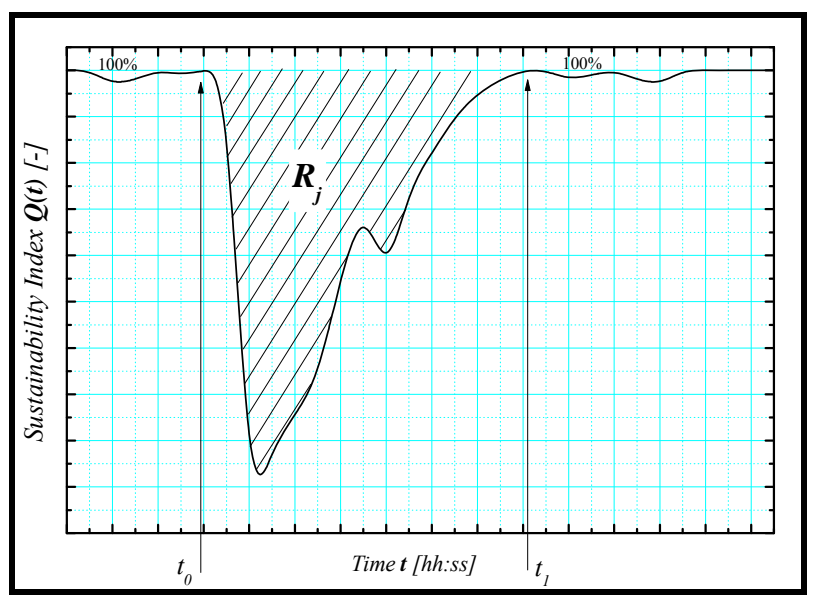

Figure 3. Resiliency index.

\section{Resilience Indicators}

In this analysis of the Resilience Index of High Voltage Transmission System a following indicators are take into a consideration, as shown on Figure 5.

\subsection{Economic Indicator}

The economic indicator are including: Electricity Cost indicator and Investment Cost. Electricity Cost indicator is representing financial loss due to electricity cut by the sudden change of the electricity cost indicator measured in the Euro/kWh. The maximum change of this indicator is estimated to $1.2 \mathrm{cEuro} / \mathrm{kWh}$.

\subsection{Environment Indicator}

It is very common that the change of environment in the vicinity of High Voltage Transmission System is affecting the power system wiring and producing the change of the ice coating affecting the wire temperature. Due to the sudden change in the wire temperature its recovery will require the time period to reach recovered state. The maximum sudden change the ice break will be $\delta /$ voltage line diameter $=0.5$.

\subsection{Social Indicators}

\subsubsection{Blackout}

Any disruption of the electric power system leads to the change of power consumption. Its effect on the power transmission to the human dwelling will affect the human life. The substitution to the power consumption deficiency is a blackout and will lead to the change of resilience index of the high voltage transmission system. It is anticipated that the maximum disruption of the electricity power system $\Delta \mathrm{v} /$ standard voltage $=20$.

\subsubsection{Human Behaviors}

It is of interest to verify human behavior related to the sudden electricity disruption. It is commonly accepted that the human reaction is measured by number of people being actively involved in the specific event. Particular attention is devoted to the effect of human behaviors during the accident if there is any. In definition human behavior it is assumed that the human effect maximum is $\Delta$ number/total number of people $=10$ being supplied by the power transmission system.

\section{Resilience Index of High Voltage Transmission Options}

Resilience engineering is applied in a number of systems 


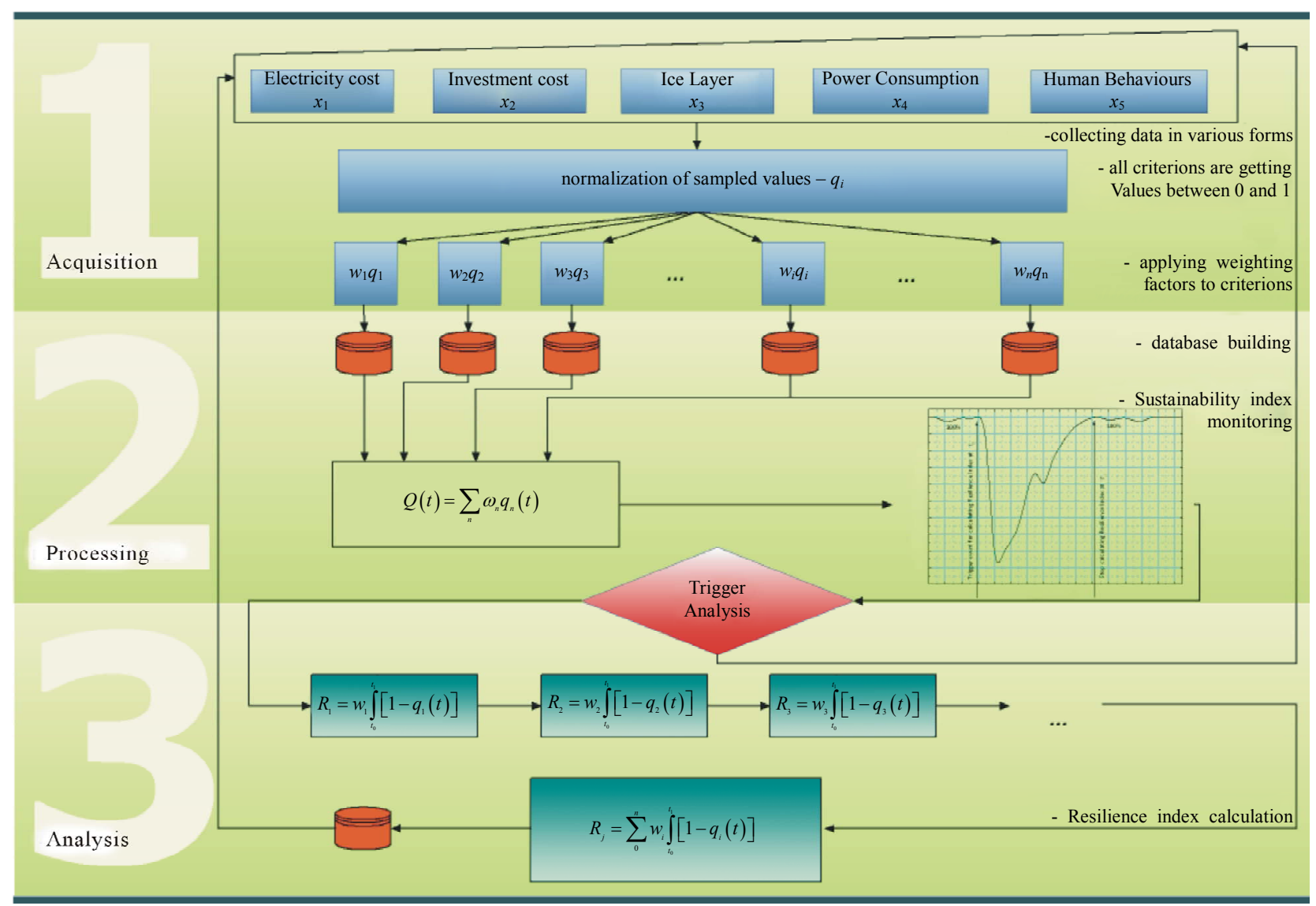

Figure 4. Resilience monitoring scheme.

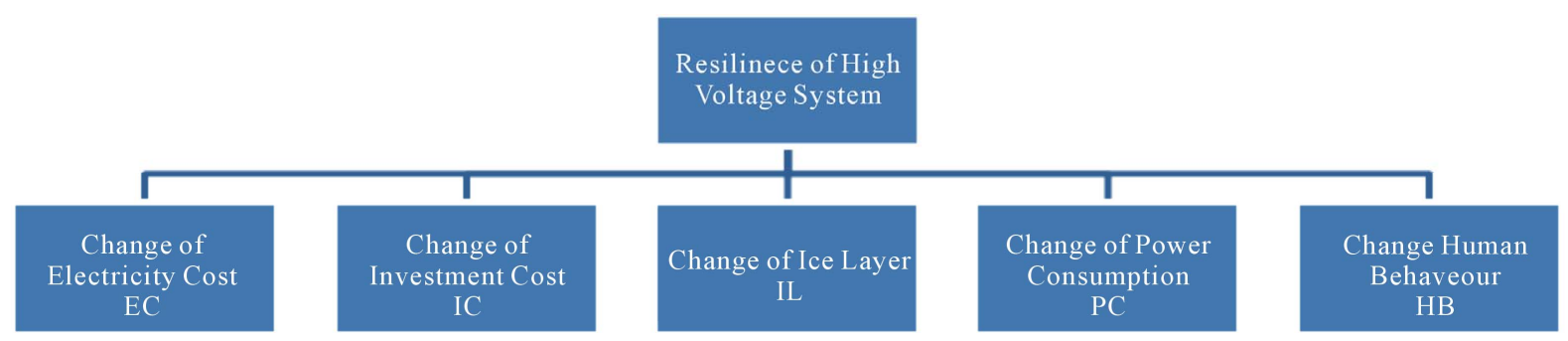

Figure 5. Agglomeration scheme of the resilience index.

in order to justify potential stability limits which may lead to the catastrophic events [14]. The resilience of the high voltage transmission system is the capacity of the system to withstand the sudden change of the internal or external parameters of the system. It reflects the quality of the system measured by the appropriate changes of the indicators. The potential possibility of the high voltage transmission system is to reach limits leading to the catastrophic events require the investigation of the cases which might be the qualitative measure of the stability of the system. As regards the high voltage transmission system a number of parameters are taken as the potential changes to be used for the verification of the individual cases.

\subsection{Options under Consideration}

In the definition of objects to be taken into a consideration it is anticipated that the high-voltage system is defined with the following parameters: Electricity Cost, Investment, Ice Layer, Change in Power Consumption, and Human Behaviour. Each of the object under consideration is defined with the set quality indicators comprising a specific value for every quality indicator. At this point it is of interest to reveal that the meanings of specific values of the quality indicators are reflecting the object description as the complex system.

As it is known the sustainability index is an appropriate parameter for the verification of the complex system. 
So, by the measurement of sustainability index under consideration the quality of the objects are verified. Since every object is defined as the specific option, we will use the change of the sustainability index due to the change of the specific indicator as the initial definition for the system definition.

In this analysis a four options are taken into a consideration with sudden change of indicators as shown in Table 1.

In this analysis a following options are taken into a consideration:

\subsubsection{Option 1-Change of Electricity Cost}

The electricity cost sub-indicator is one of the economic indicators which is subject to sudden changes due to market fluctuation. It is usually expressed in cEuro/ $\mathrm{kWh}$ reflecting the market change of the economic environment. It is anticipated to design the potential electricity cost to be expressed as $5 \mathrm{cEuro} / \mathrm{kWh}$. In this analysis the maximum the sudden change electricity cost sub-indicator is $20 \%$ of the standard electricity cost. In the design of this option we will anticipate that the changes of other indicators are participating in the definition of the object as presented in Table $\mathbf{1}$.

\subsubsection{Option 2-Change of Ice Layer}

Due to the adverse climate in the vicinity of the high voltage transmission line there is potential possibility for the formation of the ice layer on the power line wires. This ice formation will have adverse effect on the power transmission. There is potential development of the ice layer. The change of the ice layer thickness leads to the increase the weight of the ice which may cause fracture of the power line. In the design of the power transmission line special precautions is made to preserve safety of the power lines. In this respect the design of power line include the maximum thickness as of the ice layer as the limit to prevent eventual catastrophic events. In the design of this option it is anticipated that the maximum of ice thickness is $\delta / \mathrm{d}=0.5$ and other indicators will have values as presented in Table 1.

Table 1. Resilience indicators.

\begin{tabular}{ccccc}
\hline & $\begin{array}{c}\text { Electricity } \\
\text { Cost }\end{array}$ & $\begin{array}{c}\text { Ice } \\
\text { Thickness }\end{array}$ & $\begin{array}{c}\text { Public } \\
\text { Consumption }\end{array}$ & $\begin{array}{c}\text { Human } \\
\text { Behavior }\end{array}$ \\
\cline { 2 - 5 } & $\Delta \mathrm{cEURO} / \mathrm{kWh}$ & $\Delta \delta / \mathrm{d}$ & $\Delta \mathrm{kWh} / \mathrm{cap}$ & $\Delta \mathrm{N} /$ Total \\
\hline Option 1 & 1 & 0 & 50 & 5 \\
Option 2 & 0.5 & 0.5 & 0 & 2.5 \\
Option 3 & 0.25 & 0.25 & 200 & 0 \\
Option 4 & 0 & 0.125 & 100 & 10 \\
\hline
\end{tabular}

\subsubsection{Option 3-Change of Power Consumption}

The change of power consumption is an immanent problem for any high voltage transmission line. There is a possibility to have sudden increase of the power demand in some urban regions leading to the potential critical state of the power transmission. It is of interest to notify that the change in power consumption and its maximum value may result in the catastrophic event. The sudden maximum change of power consumption may lead to the catastrophic event. In the design of this option the maximum sudden change of the power consumption is $200 \mathrm{kWh} / \mathrm{cap}$. All other indicators value are given in Table 1.

\subsubsection{Option 4-Change in Human Behaviors}

The social aspect of the potential sudden change of the electric power consumption may lead to the diverse reaction of the human behavior. In particular, the prediction of the human behaviors is important issue which may lead to the catastrophic events. The human dwellings are designed with the respective communication space in order to make possible human movement within the dwelling under a severe power shortage. In the situation when it happens there is a need for mass communication. The maximum value of this indicator $\Delta \mathrm{N} /$ Total $=10$ persons/total number. For other options the values of the sudden change human behavior is given in Table 1.

\section{Case Demonstration}

In this exercise a following cases are taken into a consideration:

$\mathrm{CASE} 1-\mathrm{EC}>\mathrm{IL}=\mathrm{PC}=\mathrm{HB}$

Case 1 represent situation when the priority is given to the Change of Energy Cost indicator with other indicators having the same value, as shown on Figure 6. It is of interest to notice that if the priority given to the Change of Electricity Cost Indicator the result prove that the relation among options under consideration is having the highest value of Resilience Index with Option 1.

CASE 2- IL $>$ EC $=\mathrm{PC}=\mathrm{HB}$

The case 2 is designed with priority given to Change of Ice Layer Indicator, as shown on Figure 7. The relation among options under consideration shows a marginal difference of the resilience index among options. Priority rating in this case is: Option 2, Option 4, Option 3 and Option 1.

$\mathrm{CASE} 3-\mathrm{PC}>\mathrm{EC}=\mathrm{IL}=\mathrm{HB}$

It is of interest to notice that case 3 presents the resilience index relation for the priority given to Change of Power Consumption, as shown on Figure 8. The contribution of the other changes to the mutual relation is very similar to the other cases under consideration. 
Weighting Coefficients

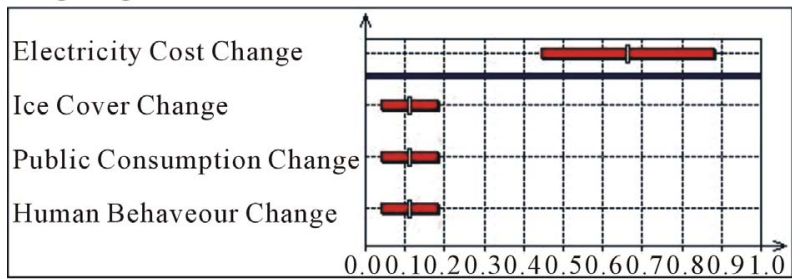

Resilience Index

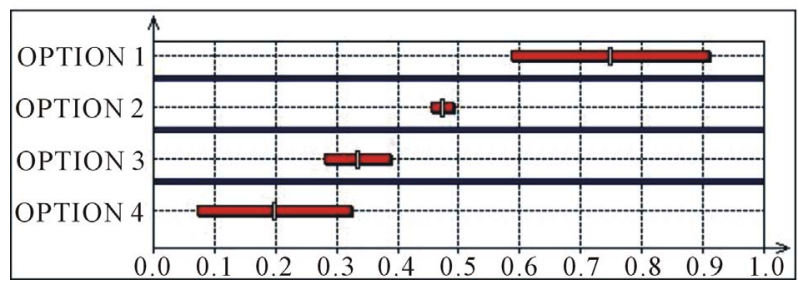

Figure 6. Resilience index-case 1.

Weighting Coefficients

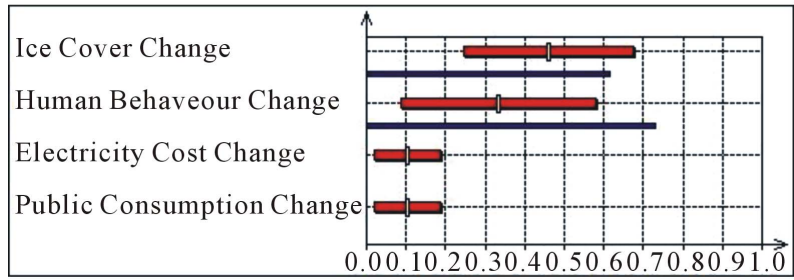

Resilience Index

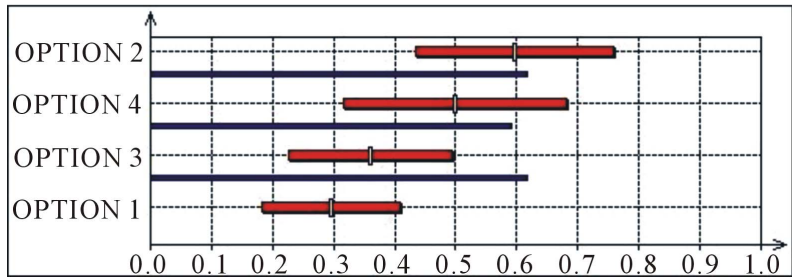

Figure 7. Resilience index-case 2.

Weighting Coefficients

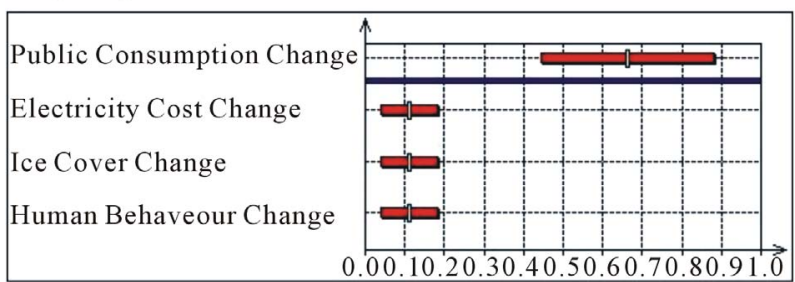

Resilience Index

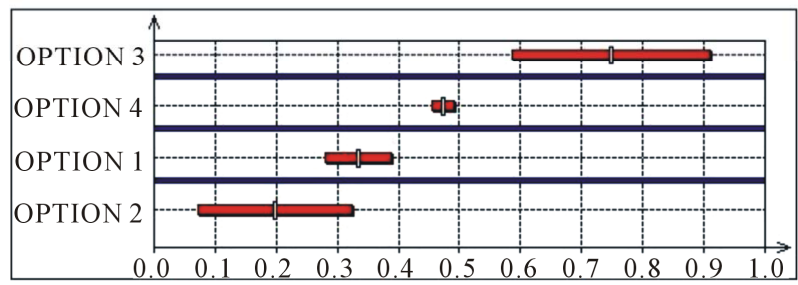

Figure 8. Resilience index-case 3.
CASE 4- $\mathrm{HB}>\mathrm{EC}=\mathrm{IL}=\mathrm{PC}$

The change in Human Behavior effect on the rating list among the options is very limited as regard resilience index for the other option, as shown on Figure 9. In this respect it is of interest to verify that the difference of the resilience index value for of options are in following rating: Option 4, Option 1, Option 2 and Option 3 as shown on Figure 9.

\section{Conclusions}

The resilience index of high voltage transmission system is the capacity to measure the stability of the system. The potential occurrence of the adverse affect is an immanent catastrophic event leading to the disruption of the high voltage structure. There are a number of the indicators which can be used for the assessment of the stability of the system. The selection of appropriate indicators is a primary goal in the design of the stability of the system. It reflects the quality of the system measured by the appropriate changes of the indicators. The potential possibility of the high voltage transmission system is to reach limits leading to the catastrophic events require the investigation of the cases which might be the qualitative measure of the stability of the system. As regards the high voltage transmission system a number of parameters is taken as the specific indicators for the definition of the potential changes to be used for the verification of the individual cases $[15,16]$.

In this analysis of the Resilience Index of High Voltage Transmission System a following indicators are take into a consideration:

\subsection{Economic Indicator}

Electricity Cost indicators is representing financial losses

Weighting Coefficients

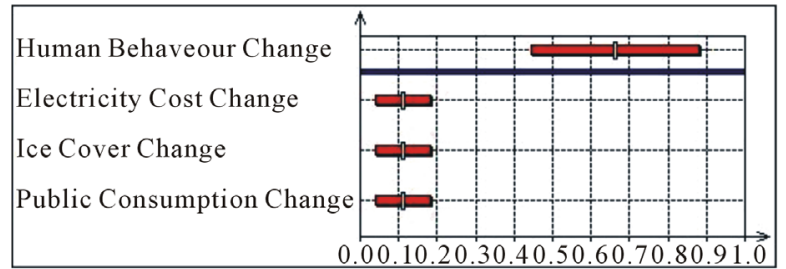

Resilience Index

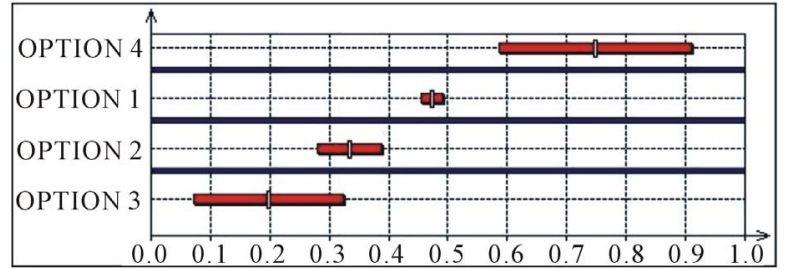

Figure 9. Resilience index-case 4. 
due to electricity cut by the sudden change of the respect- tive indicator measured in the cEuro/kWh.

\subsection{Environment Indicator}

\section{Ice Agglomeration on the High Voltage Wiring}

It is very common that the change of the environment in the vicinity of High Voltage Transmission System is affecting the power system wiring and producing the change of the ice coating affecting the wire temperature. Due to the sudden change in the wire temperature its recovery will require the time period to reach recovered state.

\subsection{Social Indicator}

\subsubsection{Blackout}

Any sudden disruption of the electricity transmission to the human dwelling will affect human life. In this respect there will be need to ensure the capacity recovery of the system if there will be any sudden change in the electricity supply

\subsubsection{Human Behaviors}

It is of interest to verify human behavior related to the sudden electricity disruption. It is commonly accepted that the human reaction is measured by number of people being actively involved in the specific event. Particular attention is devoted to the effect of human behaviors during the accident.

In general, any sudden change of the selected indicators may lead to the change of the resiliency of the system. For this reason the development of appropriate procedure for the resiliency index evaluation is a tool for the assessment of the safety of high voltage system and prevention of the catastrophic events leading to the structure destruction.

Demonstration exercise of the high voltage transmission system has been introduced as the method of verifycation of the potential limits for the catastrophic events.

\section{References}

[1] H. De Kautender and A. Boggini, "Resilience Power Supply in Modern Office Building," European Copper Insti- tute, Washington DC, June 2005.

[2] A. Chalmers and F. A. Voorvaat, "High Voltage Transmission System Lines: Proximity, Visibility, and Encumbrabce Effects," The Appraisal Journal, 2009, pp. 227 245.

[3] European Smart Grid, "Directorate-General for Research Energy System," EUR 22040, 2006.

[4] The Smart Grid, "DOE Smart Grid Pdf," USA Department of Energy, Litos Strategic Communication, Washington DC, 2008.

[5] L. Lav and J. A. G. Blagin, "Waste Case Electricity Scenario," CREATE Symposium, University of South California, Los Angeles, August 1995.

[6] W. N. Adger, "Vulnerability," Global Environment Change, Vol. 16, No. 3, 2006, pp. 268-281. doi:10.1016/j.gloenvcha.2006.02.006

[7] H. Kainan, "Concept and Practices of 'Resilience'," US Agency for International Development, Bangkok, 2006.

[8] E. Hollnagel, D. Woods and N. Levesen, "Residence Engineering: Concepts and Percepts," Ashgate Publishing Limi- ted, London, 2005.

[9] S. Atonsen, "Safety, Culture and Failure Foresight," Proceeding of the Third Resilience Engineering Symposium, Antibes, 28-30 October 2008, p. 1.

[10] C. S. Holling, "Resilience and Stability of Ecological System," Annual Review of Ecology and Systematic, Vol. 4 1973, pp. 1-23. doi:10.1146/annurev.es.04.110173.000245

[11] E. Hollnagel, P. Nemet and S. Dokker, "Resilience Engineering Perspective," Ashgate Publishing Limited, London, June 2008.

[12] Th. Priestlaym and P. C. Ignelzi, "Transmission Line Impact on Residents Communities," Elisen Electric Institute, Washington DC, June 1989.

[13] C. Folke, "Resilience: The Emergence of a Perspective for Social-Ecological System," Global Environmental Change, Vol. 16, No. 3, 2006, pp. 253-267. doi:10.1016/j.gloenvcha.2006.04.002

[14] N. Afgan and D. Cvetinovic, "Wind Power Plant Resilience," Thermal Science, Vol. 14, No. 2, 2010, pp. 533540. doi:10.2298/TSCI1002533A

[15] N. Afgan and M. G. Carvalho, "Energy System Assessment with Sustainable Indicators," Kluwer Academic Publisher, New, York, 2004.

[16] N. Afgan and M. G. Carvalho, "Quality, Sustainability and Indicators for Energy Systems," Begell House Publisher, New York, 2009. 